\title{
ABEL'S INTEGRAL EQUATION AS A CONVOLUTION TRANSFORM
}

D. B. SUMNER

1. Introduction. The Abel integral equation

$$
F(X)=\int_{0}^{X}(X-T)^{-\alpha} \Phi(T) d T \quad(0<\alpha<1, X>0),
$$

the first equation to be treated and solved as an integral equation, has an extensive literature, dealing on the one hand with properties of the functions involved, and on the other hand with the solution, and conditions for solubility of the equation. In the first category one might cite, in the modern spirit, the memoirs of Hardy [1, pp. 145$150]$ and Hardy and Littlewood [2, pp. 565-606]; and in the second category, Abel's original work [3, pp. 97-101], and the work of Tonelli [4, pp. 183-192], Tamarkin [5, pp. 219-228], Doetsch [6, pp. 192-207] and Röthe [7, pp. 375-380].

In [3], [4] and [5], the operation performed on the right hand side of (1.1) is recognized to be essentially an integration of fractional order $1-\alpha$ and the solution is obtained by making an integration of appropriate order. In Abel's memoir [3], no assumptions other than those implicitly involved in the integrations are stated about the given function $F(X)$ and the unknown function $\Phi(T)$, while the Lebesgue integral is the basis of [4] and [5]. Doetsch [6] uses the Laplace transform, and assumes $\Phi(T)$ to be continuous for $T \geqq 0$, and differentiable. In [7] the theory of the Beta function is used, and strong differentiability conditions are imposed on $F(X)$.

In the present note, equation (1.1) is treated from the point of view of the convolution transform, and an inversion operator of integro-differential type obtained for it. The Lebesgue integral is the basis of the work, but on account of the infinite integrals which occur, an additional, but relatively mild, condition is imposed on the behaviour of $\Phi(T)$ for large positive $T$.

We assume throughout that

$$
\begin{aligned}
\Phi(T) & \in L\left(0 \leqq T \leqq T_{0}\right), \text { all positive } T_{0}, \\
\int_{0}^{T} \Phi(U) d U & =O\left(T^{\alpha-\delta}\right), \quad(T \rightarrow \infty), \delta \text { positive. }
\end{aligned}
$$

Received by the editors November 11, 1954 and, in revised form, April 6, 1955. 
2. The integral equation as a convolution transform. If the substitutions $X=\exp (-x), T=\exp (-t)$ are made in (1.1), it takes the form

$$
f(x)=\int_{x}^{\infty}[\exp (t-x)-1]^{-\alpha} \phi(t) d t
$$

where $f(x)=F(\exp -x), \phi(t)=\exp [t(\alpha-1)] \Phi(\exp -t)$. This equation is a convolution transform

$$
f(x)=\int_{-\infty}^{\infty} K(x-t) \phi(t) d t,
$$

the nucleus $K(x)$ being given by

$$
K(x)= \begin{cases}{[\exp (-x)-1]^{-\alpha},} & x<0 \\ 0, & x>0 .\end{cases}
$$

It is convenient at this point to quote certain known results [8] about the generalized Stieltjes transform

$$
G(X)=\int_{0}^{\infty} \Phi(T) d T /(X+T)^{\alpha}
$$

which becomes

$$
g(x)=\int_{-\infty}^{\infty} \phi(t) d t /[1+\exp (t-x)]^{\alpha}
$$

after the above change of variables. The discussion in [8] dealt with (2.4), but we state the results in terms of (2.5).

Lemma. Let $\phi(t) \in L$ in any finite interval, and be such that the integral (2.5) converges for any complex $x_{0}$ in the strip $|\operatorname{Im} x|<\pi$, and let the principal value of $[1+\exp (t-x)]^{-\alpha}$ be taken: then the integral converges for all $x$ in the strip, and defines a function analytic in the strip. Also

$$
\begin{aligned}
(2 \pi)^{-1} \lim _{\theta \rightarrow \pi} \int_{-\theta}^{\theta}[1+\exp (-i y)]^{\alpha-1} g^{\prime}(x+i y) d y & \\
& =\frac{1}{2}[\phi(x)+\phi(x-)]
\end{aligned}
$$

whenever the right-hand side has a meaning.

3. The inversion operator. According to a fundamental result due 
to Wiener [9, pp. 557-584], one may conjecture an inversion operator $E(D)$ for equation (2.2) from

$$
1 / E(\lambda)=\int_{-\infty}^{\infty} \exp (-\lambda x) K(x) d x,
$$

where a suitable interpretation must be found for $E(D)$. On using (2.3), we find that

$$
\frac{1}{E(\lambda)}=\int_{-\infty}^{0} \frac{\exp (-\lambda x) d x}{[\exp (-x)-1]^{\alpha}}=\frac{\Gamma(1-\alpha) \Gamma(\alpha-\lambda)}{\Gamma(1-\lambda)} .
$$

Since this function is meromorphic in $\lambda$, we write

$$
E(\lambda)=\Delta(\lambda) \cdot I(\lambda)
$$

where

$$
\Delta(\lambda)=\Gamma(\alpha) / \Gamma(\lambda) \Gamma(\alpha-\lambda), \quad I(\lambda)=\sin \pi \alpha / \sin \pi \lambda .
$$

Since $\Delta(\lambda)$ is an entire function with zeros at $\lambda=\alpha, \alpha+1, \ldots$ and $\lambda=0,-1,-2, \cdots$, while $I(\lambda)$ has no zeros but poles at $\lambda=0, \pm 1$, $\pm 2, \cdots$, the operator $E(\lambda)$ is of integro-differential type, of the kind considered by Widder [10, pp. 119-128], Meijer [11, pp. 727737 and 831-839], and the author [12, pp. 114-117].

In applying the operators $I(D)$ and $\Delta(D)$, we use the representations

$$
\begin{aligned}
& I(\lambda)=\frac{\sin \pi \alpha}{\pi} \int_{-\infty}^{\infty} \frac{\exp (-\lambda v) d v}{1+\exp (-v)}, \\
& \Delta(\lambda)=\lambda(2 \pi)^{-1} \int_{-\pi}^{\pi}[1+\exp (-i y)]^{\alpha-1} \exp (i y \lambda) d y,
\end{aligned}
$$

and interpret $\exp (k D)$ as a shift operator,

$$
\exp (k D) \cdot h(x)=h(x+k),
$$

no assumption as to the differentiability of $h(x)$ being made.

4. The inversion theorem. On account of (3.4), (3.5), and (3.6) we interpret

(4.1) $I(D) \cdot f(x)=\frac{\sin \pi \alpha}{\pi} \int_{-\infty}^{\infty} \frac{f(x-v) d v}{1+\exp (-v)}$,

(4.2) $\Delta(D) \cdot g(x)=(2 \pi)^{-1} \lim _{0 \rightarrow \pi} \int_{-\theta}^{\theta}[1+\exp (-i y)]^{\alpha-1} g^{\prime}(x+i y) d y$. 
Using these interpretations, we prove the theorem:

Theorem. Let $\phi(t) \in L$ in any finite interval, let $0<\alpha<1$, and let $f(x)$ be defined by (2.1): then

$$
\begin{aligned}
I(D) \cdot f(x) & =\int_{-\infty}^{\infty} \phi(t) d t /[1+\exp (t-x)]^{\alpha} \equiv g(x) ; \\
\Delta(D) \cdot g(x) & =\frac{1}{2}[\phi(x+)+\phi(x-)],
\end{aligned}
$$

whenever the right hand side has a meaning.

On making the substitution $\exp (x-t-v)=u /(1+\lambda-\lambda u)$, where $\lambda=\exp (t-x)$, it is easily verified that

$$
\begin{aligned}
G(x, t) & \equiv \frac{\sin \pi \alpha}{\pi} \int_{x-t}^{\infty} \frac{d v}{[1+\exp (-v)][\exp (v+t-x)-1]^{\alpha}} \\
& =[1+\exp (t-x)]^{-\alpha} .
\end{aligned}
$$

From (2.1)

$$
\begin{aligned}
I(D) \cdot f(x) & =\frac{\sin \pi \alpha}{\pi} \int_{-\infty}^{\infty} \frac{d v}{[1+\exp (-v)]} \int_{x=0}^{\infty} \frac{\phi(t) d t}{[\exp (t+v-x)-1]^{\alpha}} \\
& =\int_{-\infty}^{\infty} G(x, t) \phi(t) d t,
\end{aligned}
$$

by Fubini's theorem if it is applicable. But the integral

$$
\int_{-\infty}^{\infty} G(x, t)|\phi(t)| d t
$$

converges absolutely by (1.3) when $\phi(t) \in L$ in any finite interval, and the conclusion (4.5) is therefore justified. Thus

$$
I(D) \cdot f(x)=\int_{-\infty}^{\infty} G(x, t) \phi(t) d t
$$

when $x$ is real.

From the definition of $g(x)$ above we may by (1.3) use the lemma quoted in $\$ 2$. The function $g(x+i y)$ is then analytic in the region $|y|<\pi$, and we may apply (2.6) to deduce that

$$
\Delta(D) \cdot g(x) \equiv[\phi(x+)+\phi(x-)] / 2,
$$

whenever the right hand side has a meaning. This completes the proof of our theorem. 


\section{REFERENCES}

1. G. H. Hardy, On some points of the integral calculus, Messenger of Mathematics vol. 47 (1917-1918).

2. G. H. Hardy and J. E. Littlewood, Some properties of fractional integrals, Math. Zeit. vol. 27 (1927-1928).

3. N. H. Abel, Resolution d'un problème de mécanique, Oeuvres Complètes, New ed., vol. I, section IX.

4. L. Tonelli, Sun un problema di Abel, Math. Ann. vol. 99 (1928).

5. J. D. Tamarkin, On integrable solutions of Abel's integral equation, Ann. of Math. vol. 31 (1930).

6. G. Doetsch, Integro-differentialgleichungen vom Faltungstypus, Math. Ann. vol. 89 (1923).

7. R. Röthe, Zue Abelschen Integralgleichung, Math. Zeit. vol. 33 (1931).

8. D. B. Sumner, $A n$ inversion formula for the generalized Stieltjes transform, Bull. Amer. Math. Soc. vol. 55 (1949).

9. N. Wiener, The operational calculus, Math. Ann. vol. 95 (1925-1926).

10. D. V. Widder, Symbolic inversions of the Fourier sine transform and of related transforms, J. Indian Math. Soc. vol. 14 (1950).

11. C. S. Meijer, Eine neue Erweiterung der Laplace-Transformation, Nederlandsche Akademie van Wetenschappen, Proc. Section of Sciences vol. 14 (1941).

12. D. B. Sumner, $A$ convolution transform admitting an inversion formula of integro-differential type, Canadian Journal of Mathematics vol. 5 (1953).

Research Institute of the Canadian Mathematical Congress and Hamilton College, McMaster University 